EPiC Series in Computing
Volume 70, 2020, Pages 173-181
$\begin{gathered}\text { Proceedings of the 12th International Conference } \\ \text { on Bioinformatics and Computational Biology }\end{gathered}$

\title{
Epileptic Focus Localization Based on iEEG Plot Images by Using Convolutional Neural Network
}

\author{
Xuyang Zhao ${ }^{1,2, *}$, Linfeng Sui ${ }^{1, *}$, Toshihisa Tanaka ${ }^{3,2}$, \\ Jianting $\mathrm{Cao}^{1,2}$ and Qibin Zhao ${ }^{2,4}$ \\ 1 Department of Electronic Engineering, \\ Saitama Institute of Technology, Fukaya, Japan \\ e7001gct, n8007dov, cao@sit.ac.jp \\ 2 Tensor Learning Unit, \\ RIKEN Center for Advanced Intelligence Project, Tokyo, Japan \\ qibin.zhao@riken.jp \\ 3 Department of Electrical and Electronic Engineering, \\ Tokyo University of Agriculture and Technology, Tokyo, Japan \\ tanakat@cc.tuat.ac.jp \\ 4 Ningbo Haizhi Institute of Materials Industry Innovation, Ningbo, China
}

\begin{abstract}
Patients with epilepsy need to locate the lesion before surgery. Currently, clinical experts diagnose the lesions through visual judgment. In order to reduce the workload of clinical experts, many automatic diagnostic methods have been proposed. Usually, the automatic diagnostic methods often use only one feature as the basis for diagnosis, which has certain limitations. In this paper, we use multiple feature fusion methods for automatic diagnosis. For the cause of epilepsy: abnormal discharge, we use the filter and entropy to capture the energy features of epilepsy discharge. Due to the epilepsy brain waves contain spike and shape waveforms, short time Fourier transform (STFT) is used to analysis the time-frequency features. In feature fusion, we plot the color map of entropy and spectrogram get from STFT together to combine the different types of features. After the feature extraction and fusion steps, each brain signal is converted into an image. Next, we use the visual analysis capabilities of the convolutional neural network (CNN) to classify the plot image. With the visual recognition ability of $\mathrm{CNN}$, in the experiment, we got a classification accuracy of $88.77 \%$. By using automatic diagnostic methods, the workload of clinical experts is greatly reduced in actual clinical practice.
\end{abstract}

\section{Introduction}

Epilepsy is a brain disease caused by abnormal discharges of brain cells. According to the World Health Organization (WTO) statistics, approximately 50 million people worldwide have epilepsy. Epilepsy disease brings a variety of social problems, such as patients need long-term

* Authors contributed equally

Q. Ding, O. Eulenstein and H. Al-Mubaid (eds.), BICOB 2020 (EPiC Series in Computing, vol. 70), pp. $173-181$ 
medication, some pediatric patients will face the chilean development, neurological diseases and other problems during their growth. At present, epilepsy patients can control the seizures by taking medications, and some patients can reach a complete cure and stop taking medications. Some patients require long-term medication to control their condition. But there are still some patients with drug resistance. For this part of the patient, the current plan is to remove the lesion by surgery.

Before epilepsy surgery, we need to determine the location of the lesion through physical examination. Intracranial Electroencephalography (iEEG) is the most commonly used examination method. In current clinical practice, iEEG signal is analyzed by a clinical expert's visual diagnosis [1]. Here are some points that need improvement. iEEG recording time is at least two-three days, usually one week. These massive amounts of iEEG data require manual visual diagnosis by clinical experts. Vision diagnosis is an experience dependent process and often requires several years of training. Nevertheless, the diagnostic results given by different experts are not completely consistent, final diagnosis often required by a vote of the diagnostic committee.

In view of the problems and difficulties in the diagnosis process, some automatic diagnosis systems have been proposed. These methods mainly include two steps, feature extraction and classification. In feature extraction, wavelet transform [2, 3, 4, 5] empirical mode decomposition (EMD) [6, 7], entropy [8, 9, 10] time-frequency [11, 12] methods are often be used. In classification work, from traditional classifiers support vector machine (SVM) to current neural network based methods EEGNet [13] are often used

At present, the diagnosis of clinical expert in hospitals is often performed in a variety of ways, visual judgment of brain waves at different scales and frequency domain analysis. However, in the proposed automatic diagnosis methods, a single feature is mainly used, which has limitations on the model. In this paper, we use two different features and fuse the features together to form a new feature image. By this way, two features are combined. Time-series brain signals are converted into pictures, so we can use CNN models for visual recognition. In the feature extraction method, we start from the point that energy is contained in the over discharge, and select a variety of entropy as the features. When clinical experts make visual judgments, they often make judgments based on whether the brain waves contain spike waves and shape waves. Brain waves of healthy people are mainly composed of four wave bands: $\delta: 0.5-4 \mathrm{~Hz}$, adult slow-wave sleep and baby. $\theta: 4-8 \mathrm{~Hz}$, drowsiness in adults and teens. $\alpha$ : $8-13 \mathrm{~Hz}$, relaxed/reflecting. $\beta: 13-30 \mathrm{~Hz}$, focus, high alert, anxious and so on. The frequency range of spike wave is $14-50 \mathrm{~Hz}$ and the frequency range of shape wave is $5-14 \mathrm{~Hz}$. According to the difference in frequency distribution between normal brain wave and spike, shape wave, we use short time Fourier transform to analyze the frequency domain information. During feature fusion, we choose to fuse two different features into a image, so that we can use the powerful visual recognition capabilities of CNN

The rest of the article is organized as follows: Section 2 describes the feature extraction method include entropy \& filter, STFT and plot images. Section 3 describes the CNN model used as a classifier. The results are presented in Section 4, The discussion and summary are described in Section 5.

\section{Methods}

We start with the pathogenesis of epilepsy and the characteristics of the brain wave, use two different feature extraction methods of entropy and STFT, and fuse the two features into one image. By fusing two features into an image, we convert the brain wave signal into an image, so 
we can use the powerful classification capabilities of a convolutional neural network to classify the brain wave.

\section{$2.1 \quad$ Dataset}

Bern Barcelona dataset [14] is used to evaluate our method. The dataset is built by the Department of Information and Communication Technologies of the Universitat Pompeu Fabra and the Department of Neurology of the University of Bern. The dataset includes five patients and each patient has long standing pharmacoresistant temporal lobe epilepsy and is a candidate for epilepsy surgery. The iEEG data is recorded by using the device of AD-TECH (Racine, WI, USA). A total of 15,000 samples, 7,500 focal samples and 7,500 non-focal samples. each sample is 20 seconds with a sampling rate of $512 \mathrm{~Hz}$ and filtered by a bandpass filter $(0.5-150 \mathrm{~Hz}$, fourth-orders Butterworth filter). You can find more information on the website ${ }^{1}$. An example of the focal and non-focal iEEG samples are shown in Fig. 1, respectively.

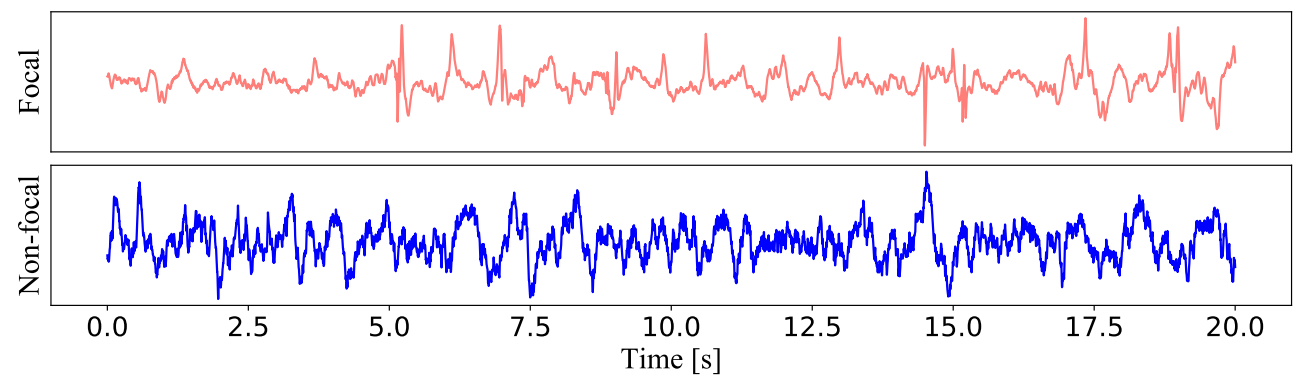

Figure 1: Samples of focal and non-focal iEEG (Bern Barcelona Dataset).

\subsection{Entropy \& Filter}

Consider the cause of epileptic seizures: abnormal discharge of brain cells. The abnormal discharge releases a lot of energy, and we use entropy [15] as a feature extraction method and combined with the filter to extract the features of the iEEG signal. First, we use six bandpass filters (third-order Butterworth) to filter the iEEG data. The bandpass frequencies used are Delta 0.5-4 Hz, Theta 4-8 Hz, Alpha 8-13 Hz, Beta 13-30 Hz, Gamma 30-80 Hz and Ripple 80$150 \mathrm{~Hz}$. After filtering, we calculate eight different entropies for each filtered iEEG data. Eight different entropies are Shannon entropy, Renee entropy, Generalized entropy [16] [17], Phase entropy (two types) [18], Approximate entropy [19], Sample entropy [20] and Permutation entropy [21] After these two steps, we extract a feature matrix from each sample with a size of $6 \times 8$. The flowchart of feature extraction procedure is shown in Fig. 2 .

\subsection{Short Time Fourier Transform}

When clinical experts diagnose epilepsy, they mainly judge whether the brain waves contain waveforms such as spike and shape waves from the visual point of view. Usually, the frequency of shape is between $5-14 \mathrm{~Hz}$ and the spike wave is between 14-50 Hz. Because of the instability

\footnotetext{
${ }^{1} \mathrm{http}$ ///ntsa.upf.edu/downloads/andrzejak-rg-schindler-k-rummel-c-2012-nonrandomness-nonlineardependence-and
} 


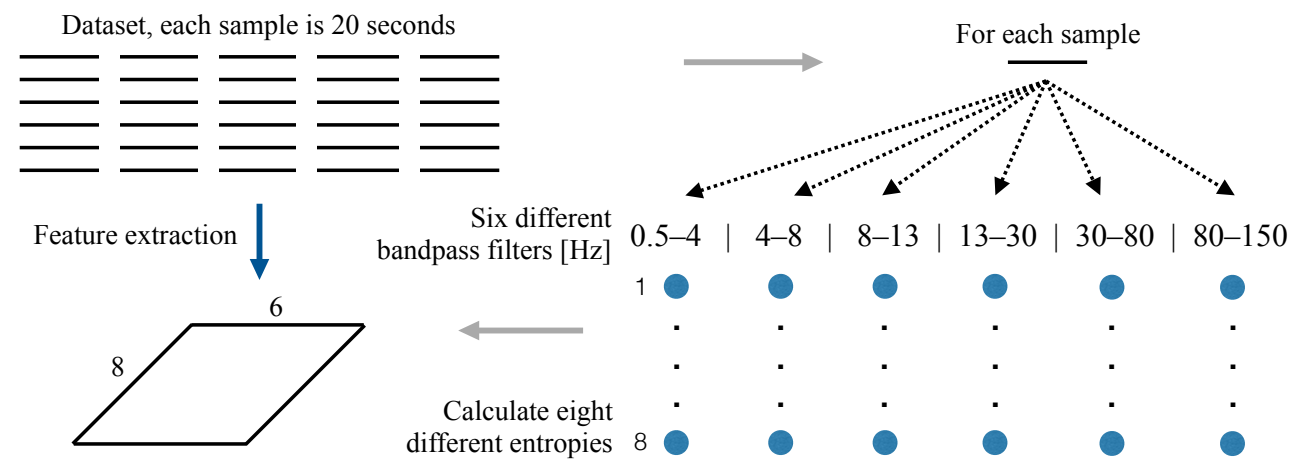

Figure 2: Flowchart of feature extract procedure: Filter \& Entropy.

of the iEEG signal, we use the STFT method to replace the Fourier transform [22] for the feature extraction. $x(t)$ is a timing signal, the time-frequency spectrogram can be calculate by (1)

$$
\operatorname{STFT}\{x(t)\}(\tau, \omega)=\int_{-\infty}^{\infty} x(t) w(t-\tau) e^{-j \omega t} d t
$$

where $w(t)$ is the Hann window function centered around zero.

\subsection{Image Plot}

In order to use the visual method to classify the extracted data features. We plot the entropy and spectrogram obtained by the STFT into an image with $224 \times 224$ pixels. The example of feature fusion image is shown in Fig. 3, images are randomly selected from the dataset.
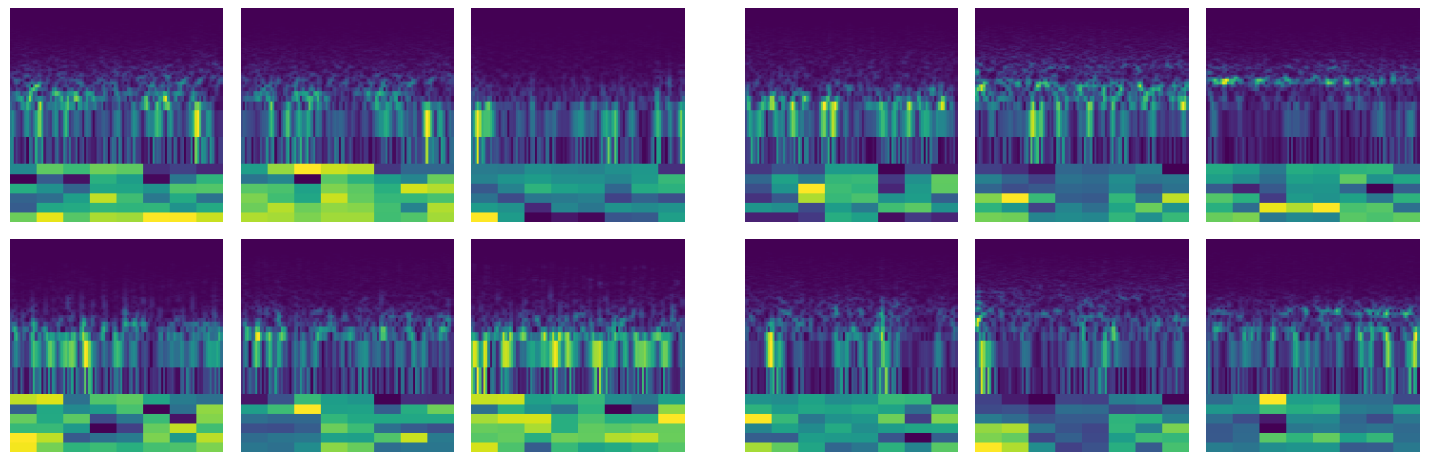

Figure 3: Each image contains two parts, the upper part is the spectrum obtained by STFT, and the lower part is drawn by the feature matrix (color map). The left half is the focal signal, and the other half is non-focal signals. 


\section{Convolutional Neural Network}

The ultimate obtained STFT \& Entropy images are fed into CNN model shown as Fig. 4. The proposed CNN architecture has four convolutional layers following three fully connected neural network (FCNN) layers, a max-pooling and a batch normalization layer are set after each convolutional layer to lower the calculation complexity and prevent overfitting. The last layer of the architecture to execute the classification process, in this layer, the input is classified as focal or non-focal.

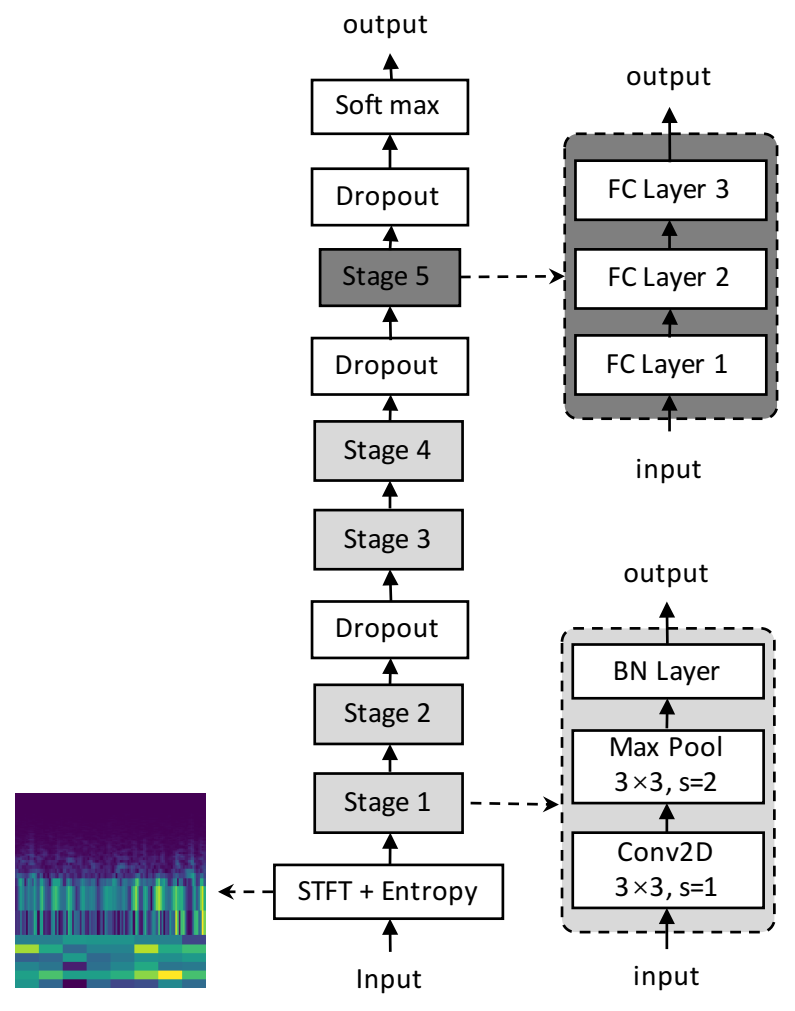

Figure 4: Architecture of CNN model.

\section{Experimental Results}

In this section, Bern Barcelona dataset is used to evaluate our method. In the dataset, a total of 15,000 samples, focal and non-focal has 7,500 samples, respectively. We use a 10-fold crossvalidation method to evaluate the model. Every time, $10 \%$ data are selected as a test set, the rest are training sets, at last, every data has been tested once.

As a comparison, we only use entropy feature with a three layers fully connected neural network model, the result is shown in Fig. 5. Another model for comparison is STFT feature with one layer of maxpool and five layers of fully connected neural network, each fully connected layer is followed by a batch normalization layer, the result is shown in Fig. 6. The result of fused features with FCNN model is shown in Fig. 7. The result of fused features with CNN 
model is shown in Fig. 8. Comparison of the results of the three models are shown in Table 1.
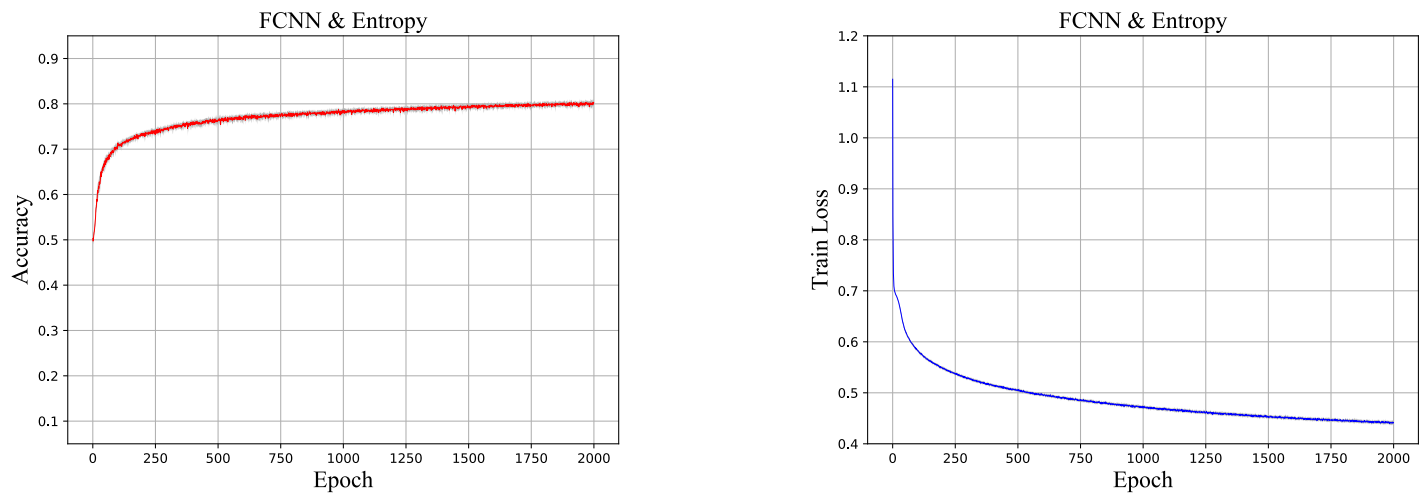

Figure 5: Results of the FCNN model with entropy features. Left: test accuracy vs. number of epochs, red line: Average of classification test accuracy (10-folds), gray area: Standard deviation. Right: Train loss vs. number of epochs, blue line: Average of train loss (10-folds), gray area: Standard deviation.
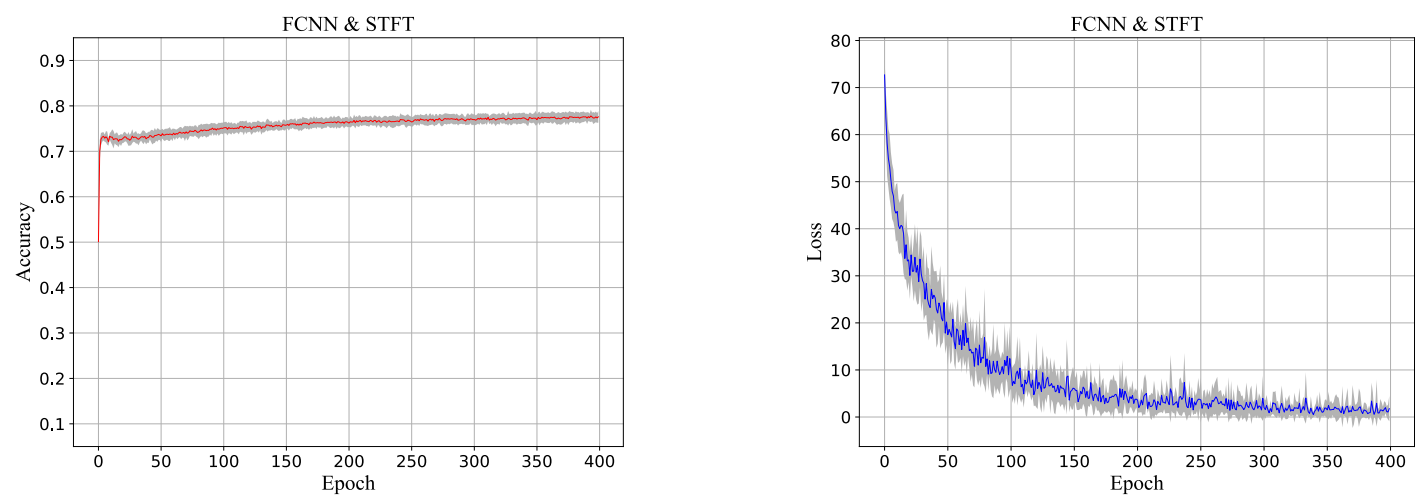

Figure 6: Results of the FCNN model with STFT features. Left: test accuracy vs. number of epochs, red line: Average of classification test accuracy (10-folds), gray area: Standard deviation. Right: Train loss vs. number of epochs, blue line: Average of train loss (10-folds), gray area: Standard deviation.

\section{Discussion and Summary}

In the article, we proceed from the principle of epilepsy disease, use entropy and short-time Fourier for feature extraction, which corresponds to the abnormal discharge (energy) and spike, shape wave (frequency characteristics) of epilepsy brain signals, respectively. In the traditional method, the extracted feature matrix is usually input into a classifier. In this paper, we plot the extracted feature matrix into an image and then use a convolutional neural network for classification. By this way, we can use the visual analysis capabilities of the CNN model. 

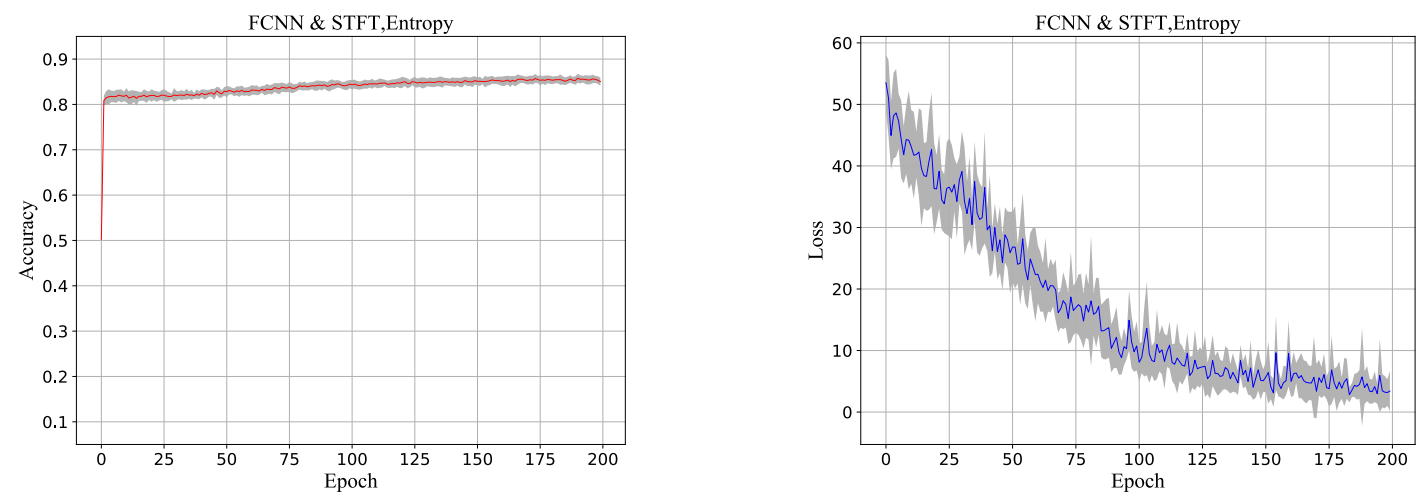

Figure 7: Results of the FCNN model with plot image. Left: test accuracy vs. number of epochs, red line: Average of classification test accuracy (10-folds), gray area: Standard deviation. Right: Train loss vs. number of epochs, blue line: Average of train loss (10-folds), gray area: Standard deviation.
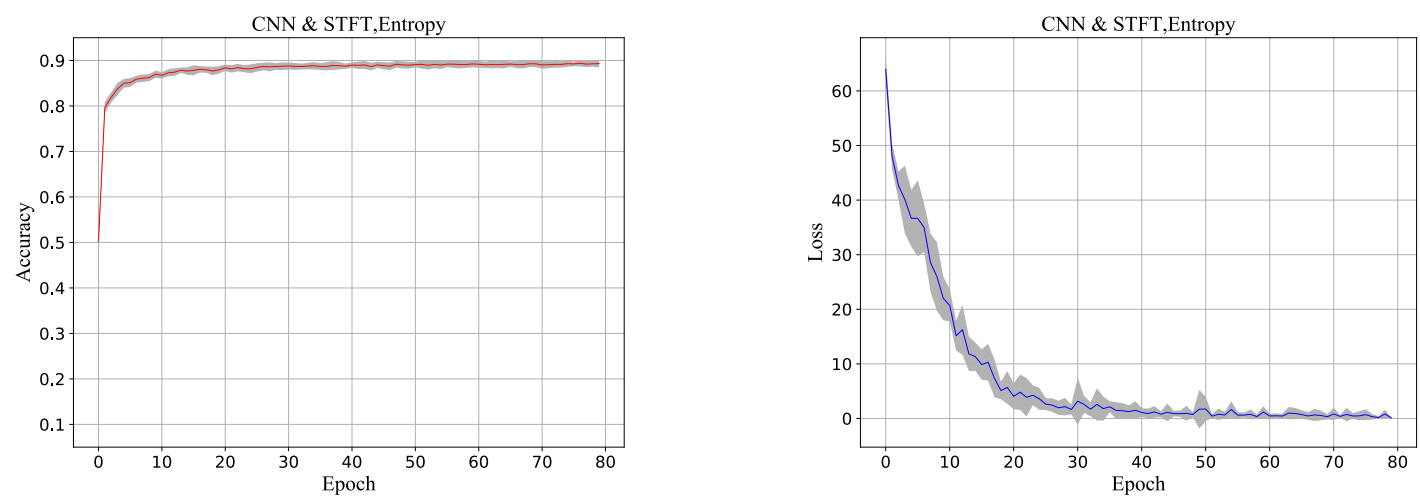

Figure 8: Results of the CNN model with plot image. Left: test accuracy vs. number of epochs, red line: Average of classification test accuracy (10-folds), gray area: Standard deviation. Right: Train loss vs. number of epochs, blue line: Average of train loss (10-folds), gray area: Standard deviation.

Comparison of algorithm performance with other articles are shown in Table 2. The results show that the performance of the model is improved by multi-feature fusion.

Table 1: Results of three models (Mean \pm standard deviation).

\begin{tabular}{ccccc}
\hline \multirow{2}{*}{ Model } & $\begin{array}{c}\text { FCNN } \\
\text { Entropy }\end{array}$ & $\begin{array}{c}\text { FCNN } \\
\text { STFT }\end{array}$ & $\begin{array}{c}\text { FCNN } \\
\text { Plot Image }\end{array}$ & $\begin{array}{c}\text { CNN } \\
\text { Plot Image }\end{array}$ \\
\hline Result [\%] & $80.06 \pm 0.16$ & $78.14 \pm 0.11$ & $85.47 \pm 0.19$ & $\mathbf{8 8 . 7 7} \pm 0.16$ \\
\hline
\end{tabular}


Table 2: Localization results of focal and non-focal iEEG data of published articles by using the Bern-Barcelona dataset (accuracy in [\%]).

\begin{tabular}{c|c|c}
\hline Author & Method proposed & Performance \\
\hline$[5]$ & SVM \& DWT & 83.07 \\
\hline$[3]$ & LS-SVM \& EMD, Entropy & 87 \\
\hline$[23]$ & LS-SVM \& DWT, Entropy & 84 \\
\hline$[9]$ & LS-SVM \& TQWT, Entropy & 84.67 \\
\hline$[6]$ & SVM \& BEMD & 86.89 \\
\hline Proposed & CNN \& Plot Image & $\mathbf{8 8 . 7 7} \pm 0.16$ \\
\hline
\end{tabular}

\section{Acknowledgments}

This work was supported by JST CREST (Grant Number JPMJCR1784), JSPS KAKENHI (Grant No. 17K00326 and 18K04178).

\section{References}

[1] Ali Hossam Shoeb. Application of machine learning to epileptic seizure onset detection and treatment. PhD thesis, Massachusetts Institute of Technology, 2009.

[2] Yusuf Uzzaman Khan, Nidal Rafiuddin, and Omar Farooq. Automated seizure detection in scalp EEG using multiple wavelet scales. In 2012 IEEE International Conference on Signal Processing, Computing and Control, pages 1-5. IEEE, 2012.

[3] Rajeev Sharma, Ram Bilas Pachori, and U Rajendra Acharya. Application of entropy measures on intrinsic mode functions for the automated identification of focal electroencephalogram signals. Entropy, 17(2):669-691, 2015.

[4] Oliver Faust, U Rajendra Acharya, Hojjat Adeli, and Amir Adeli. Wavelet-based EEG processing for computer-aided seizure detection and epilepsy diagnosis. Seizure, 26:56-64, 2015.

[5] Duo Chen, Suiren Wan, and Forrest Sheng Bao. Epileptic focus localization using EEG based on discrete wavelet transform through full-level decomposition. In Machine Learning for Signal Processing (MLSP), 2015 IEEE 25th International Workshop on, pages 1-6. IEEE, 2015.

[6] Tatsunori Itakura and Toshihisa Tanaka. Epileptic focus localization based on bivariate empirical mode decomposition and entropy. In Asia-Pacific Signal and Information Processing Association Annual Summit and Conference (APSIPA ASC), 2017, pages 1426-1429. IEEE, 2017.

[7] Anindya Bijoy Das and Mohammed Imamul Hassan Bhuiyan. Discrimination and classification of focal and non-focal EEG signals using entropy-based features in the EMD-DWT domain. Biomedical Signal Processing and Control, 29:11-21, 2016.

[8] Guohun Zhu, Yan Li, Peng Paul Wen, Shuaifang Wang, and Min Xi. Epileptogenic focus detection in intracranial EEG based on delay permutation entropy. In AIP conference proceedings, pages 31-36. AIP, 2013.

[9] Abhijit Bhattacharyya, Ram Pachori, Abhay Upadhyay, and U Acharya. Tunable-Q wavelet transform based multiscale entropy measure for automated classification of epileptic eeg signals. Applied Sciences, 7(4):385, 2017. 
[10] Forrest Sheng Bao, Jue-Ming Gao, Jing Hu, Donald YC Lie, Yuanlin Zhang, and KJ Oommen. Automated epilepsy diagnosis using interictal scalp eeg. In 2009 Annual International Conference of the IEEE Engineering in Medicine and Biology Society, pages 6603-6607. IEEE, 2009.

[11] Lotfi Senhadji and Fabrice Wendling. Epileptic transient detection: wavelets and time-frequency approaches. Neurophysiologie Clinique/Clinical Neurophysiology, 32(3):175-192, 2002.

[12] Pushpendra Singh and Ram Bilas Pachori. Classification of focal and nonfocal eeg signals using features derived from fourier-based rhythms. Journal of Mechanics in Medicine and Biology, 17(07):1740002, 2017.

[13] Vernon J Lawhern, Amelia J Solon, Nicholas R Waytowich, Stephen M Gordon, Chou P Hung, and Brent J Lance. Eegnet: a compact convolutional neural network for eeg-based brain-computer interfaces. Journal of neural engineering, 15(5):056013, 2018.

[14] Ralph G Andrzejak, Kaspar Schindler, and Christian Rummel. Nonrandomness, nonlinear dependence, and nonstationarity of electroencephalographic recordings from epilepsy patients. Physical Review E, 86(4):046206, 2012.

[15] Tatsunori Itakura, Ito Shintaro, Toshihisa Tanaka, and Sugano Hidenori. Effective frequency bands and features for epileptic focus detectionfrom interictal electrocorticogram. TECHNICAL REPORT OF IEICE, pages 311-316, 2018.

[16] N Kannathal, Min Lim Choo, U Rajendra Acharya, and PK Sadasivan. Entropies for detection of epilepsy in EEG. Computer Methods and Programs in Biomedicine, 80(3):187-194, 2005.

[17] Jikai Chen and Guoqing Li. Tsallis wavelet entropy and its application in power signal analysis. Entropy, 16(6):3009-3025, 2014.

[18] Chrysostomos L Nikias and Jerry M Mendel. Signal processing with higher-order spectra. IEEE Signal Processing Magazine, 10(3):10-37, 1993.

[19] Steven M Pincus. Approximate entropy as a measure of system complexity. Proceedings of the National Academy of Sciences, 88(6):2297-2301, 1991.

[20] Joshua S Richman and J Randall Moorman. Physiological time-series analysis using approximate entropy and sample entropy. American Journal of Physiology Heart and Circulatory Physiology, 278(6):H2039-H2049, 2000.

[21] Christoph Bandt and Bernd Pompe. Permutation entropy: a natural complexity measure for time series. Physical Review Letters, 88(17):174102, 2002.

[22] Alexandros T Tzallas, Markos G Tsipouras, and Dimitrios I Fotiadis. Epileptic seizure detection in eegs using time-frequency analysis. IEEE transactions on information technology in biomedicine, 13(5):703-710, 2009.

[23] Rajeev Sharma, Ram Bilas Pachori, and U Rajendra Acharya. An integrated index for the identification of focal electroencephalogram signals using discrete wavelet transform and entropy measures. Entropy, 17(8):5218-5240, 2015. 